\title{
Analytical Solution of Nonlinear Dynamics of a Self-Igniting Reaction-Diffusion System Using Modified Adomian Decomposition Method
}

\author{
Felicia Shirly Peace, ${ }^{1}$ Narmatha Sathiyaseelan, ${ }^{1}$ and Lakshmanan Rajendran ${ }^{2}$ \\ ${ }^{1}$ Department of Mathematics, Lady Doak College, Madurai, Tamil Nadu 625002, India \\ ${ }^{2}$ Department of Mathematics, Madura College, Madurai, Tamil Nadu 625011, India \\ Correspondence should be addressed to Lakshmanan Rajendran; raj_sms@rediffmail.com
}

Received 24 September 2013; Revised 18 December 2013; Accepted 23 January 2014; Published 24 April 2014

Academic Editor: Jean-Pierre Corriou

Copyright ( $) 2014$ Felicia Shirly Peace et al. This is an open access article distributed under the Creative Commons Attribution License, which permits unrestricted use, distribution, and reproduction in any medium, provided the original work is properly cited.

A mathematical model of the dynamics of the self-ignition of a reaction-diffusion system is studied in this paper. An approximate analytical method (modified Adomian decomposition method) is used to solve nonlinear differential equations under steadystate condition. Analytical expressions for concentrations of the gas reactant and the temperature have been derived for Lewis number (Le) and parameters $\beta, \gamma$, and $\phi^{2}$. Furthermore, in this work, the numerical simulation of the problem is also reported using MATLAB program. An agreement between analytical and numerical results is noted.

\section{Introduction}

Nonlinear dynamical phenomena in combustion process are an active area of experimental and theoretical research. Mathematical models that describe this phenomenon can be considered as nonlinear dynamical systems. The dynamic characterization of such models was developed by Continillo et al. [1]. The detailed numerical simulation of autoignition of coal stockpiles leads to the observation of steady regimes. To better investigate this phenomenon, two simplified distributed-parameter models were discussed which incorporate heat conduction, mass diffusion, and one-step Arrhenius exothermic chemical reaction. Both model equations were solved with straight forward finite-difference schemes [2]. The problem of spontaneous ignition of coal stockpiles is challenging for safety implications and for its theoretical complexity: a spontaneous combustion reaction takes place in a bed of solid fuel, while flow, driven by natural convection generated by the onset of temperature gradients within the pile, occurs. Coal stockpiles self-ignite when reaction of coal with oxygen present in the atmosphere generates heat, that is, not efficiently removed toward the external ambient [3]. Continillo et al. [4,5] have analyzed the self-combustion of coal piles in the absence of natural convection. The three main phenomena in the self-ignition of coal stockpile are convection, reaction, and diffusion. On the other hand, Continillo et al. [6] studied the dynamic behavior of a two-dimensional coal pile also by accounting for natural convection. As part of a comprehensive study of self-heating of coal stockpiles, a simple mathematical model has been developed. To our knowledge, no rigorous analytical expressions of gas reactant $(Y)$ and temperature $(T)$ have been derived for all possible values of parameters under steady-state conditions. The purpose of this paper is to derive approximate analytical expressions for gas reactant concentration and temperature using the modified Adomian decomposition method.

\section{Mathematical Formulation of the Boundary Value Problem}

The nonlinear differential equations are those of a distributed-parameter dynamic model of heterogeneous reaction in a one-dimensional layer. The gaseous reactant diffuses through the reacting medium and a first order one-step exothermic 
chemical reaction takes place. The reaction rate depends on the temperature through the Arrhenius exponential. The Arrhenius rate equation is a mathematical expression which relates the rate constant of a chemical reaction to the exponential value of the temperature. The model nonlinear equations in dimensionless form are [1]

$$
\begin{aligned}
& \frac{\partial Y}{\partial t}=\operatorname{Le} \frac{\partial^{2} Y}{\partial x^{2}}-\phi^{2} Y \exp \left(-\frac{\gamma}{T}\right), \\
& \frac{\partial T}{\partial t}=\frac{\partial^{2} T}{\partial x^{2}}+\beta \phi^{2} Y \exp \left(-\frac{\gamma}{T}\right),
\end{aligned}
$$

where $Y$ is the concentration of the gas reactant, $T$ is the temperature, Le is the Lewis number (the ratio between mass and heat diffusivities), $\beta$ is the dimensionless heat of reaction, $\phi$ is the thermal Thiele modulus (the ratio of the time scale of the limiting transport mechanism to the time scale of intrinsic reaction kinetics [7]), and $\gamma$ is the dimensionless activation energy (minimum amount of energy between reactant molecules for effective collisions between them). The boundary conditions are

$$
\begin{gathered}
T(0, t)=T(1, t)=1, \quad Y(0, t)=1, \\
\left.\frac{\partial Y}{\partial x}\right|_{x=1}=0 \quad \text { for } t>0 .
\end{gathered}
$$

Under steady-state condition, the equations become

$$
\begin{aligned}
& \frac{d^{2} Y}{d x^{2}}-\frac{\phi^{2}}{\mathrm{Le}} Y \exp \left(-\frac{\gamma}{T}\right)=0, \\
& \frac{d^{2} T}{d x^{2}}+\beta \phi^{2} Y \exp \left(-\frac{\gamma}{T}\right)=0
\end{aligned}
$$

with boundary conditions

$$
\begin{aligned}
& T=1, \quad Y=1, \quad \text { at } x=0, \\
& T=1, \quad \frac{d Y}{d x}=0 \quad \text { at } x=1 .
\end{aligned}
$$

\section{Analytical Solution of Nonlinear Dynamics of a Self-Igniting Reaction-Diffusion System under Steady-State Condition Using Modified Adomian Decomposition Method}

In the recent years, much attention is devoted to the application of the Adomian decomposition method to the solution of various scientific models [8]. An efficient modification of the standard Adomian decomposition method for solving initial value problem in the second order partial differential equation yields the MADM. The MADM without linearization, perturbation, transformation, or discretization gives an analytical solution in terms of a rapidly convergent infinite power series with easily computable terms. The results show that the rate of convergence of modified Adomian decomposition method is higher than standard Adomian decomposition method [9-13]. Using this method (see Appendix A), we

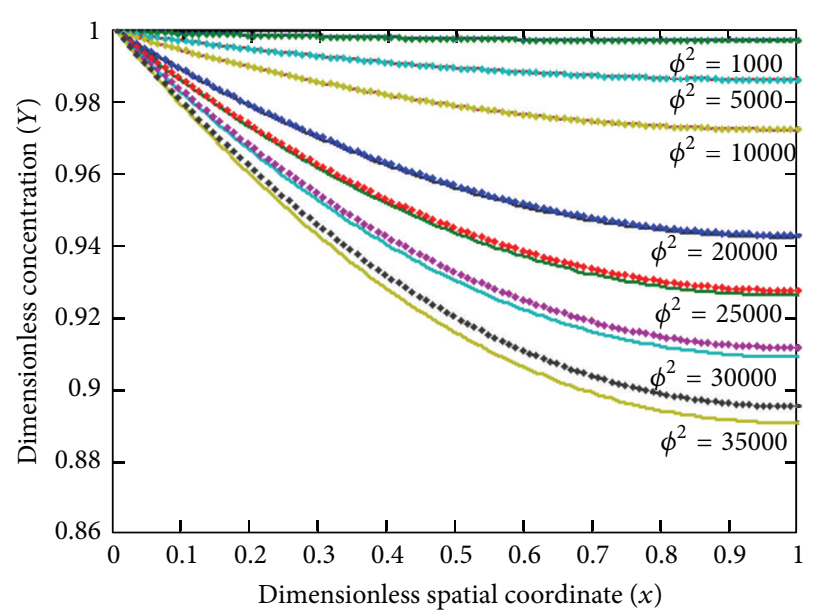

FIGURE 1: Dimensionless concentration $Y$ versus dimensionless spatial coordinate $x$ using (5) for Le $=0.233, \beta=4.287, \gamma=13.6$, and various values of $\phi^{2}$. Solid lines represent the analytical solution, whereas the dotted lines are for the numerical solution.

obtain an approximate analytical expression of concentration of gas reactant $(Y)$ and temperature $(T)$ (see Appendix B) as follows:

$$
\begin{aligned}
Y(x)= & 1+\frac{\phi^{2}}{\operatorname{Le}} \exp (-\gamma)\left(\frac{x^{2}}{2}-x\right)+\frac{\phi^{4}}{\operatorname{Le}} \exp (-2 \gamma) \\
& \times\left[\frac{1}{\operatorname{Le}}\left(\frac{x^{4}}{24}-\frac{x^{3}}{6}+\frac{x}{3}\right)-\frac{\gamma \beta}{2}\left(\frac{x^{4}}{12}-\frac{x^{3}}{6}+\frac{x}{6}\right)\right] \\
T(x)= & 1-\beta \phi^{2} \exp (-\gamma)\left(\frac{x^{2}}{2}-\frac{x}{2}\right)-\beta \phi^{4} \exp (-2 \gamma) \\
& \times\left[\frac{1}{\operatorname{Le}}\left(\frac{x^{4}}{24}-\frac{x^{3}}{6}+\frac{x}{8}\right)-\frac{\gamma \beta}{2}\left(\frac{x^{4}}{12}-\frac{x^{3}}{6}+\frac{x}{12}\right)\right]
\end{aligned}
$$

\section{Numerical Simulation}

Nonlinear diffusion equation (3) for the boundary condition (4) is also solved numerically. We have used the function pdexl in MATLAB software to solve the initial-boundary value problems for the nonlinear differential equations numerically. This numerical solution is compared with our analytical results in Figures 1-4. Upon comparison, it gives a satisfactory agreement for all values of the dimensionless parameters $\beta, \gamma$, and $\phi^{2}$. The MATLAB program is also given in Algorithm 1.

\section{Discussion}

Equations (5) and (6) represent the simplest form of approximate analytical expressions for the concentration of gas reactant and temperature for all values of parameters $\alpha$, $\beta, \gamma$, and $\phi^{2}$. The Thiele number (thermal) is the ratio of layer thickness $(L)$ and thermal diffusivity $(\alpha)$. Equation (5) 


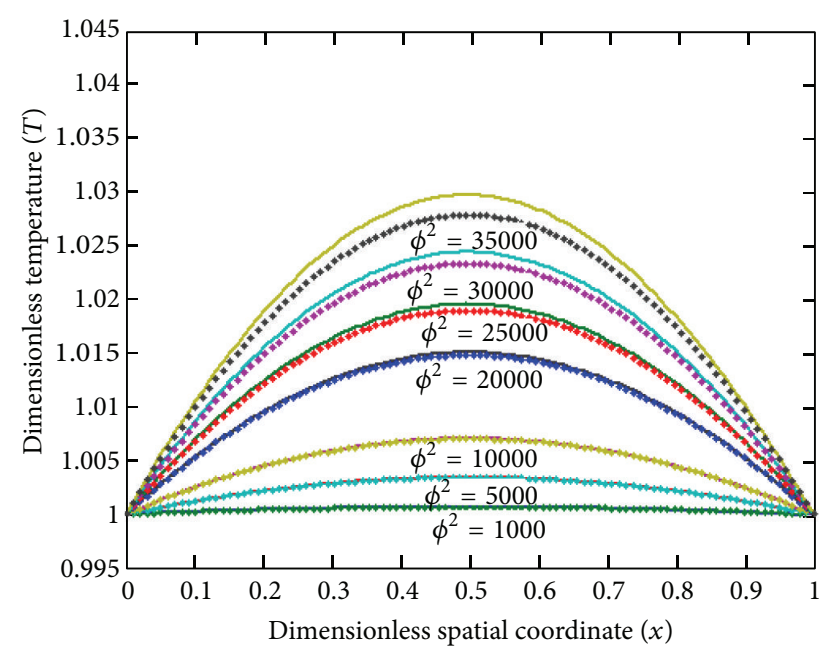

Figure 2: Dimensionless temperature $T$ versus dimensionless spatial coordinate using (6) for $\mathrm{Le}=0.233, \beta=4.287, \gamma=13.6$, and various values of $\phi^{2}$. Solid lines represent the analytical solution, whereas the dotted lines are for the numerical solution.

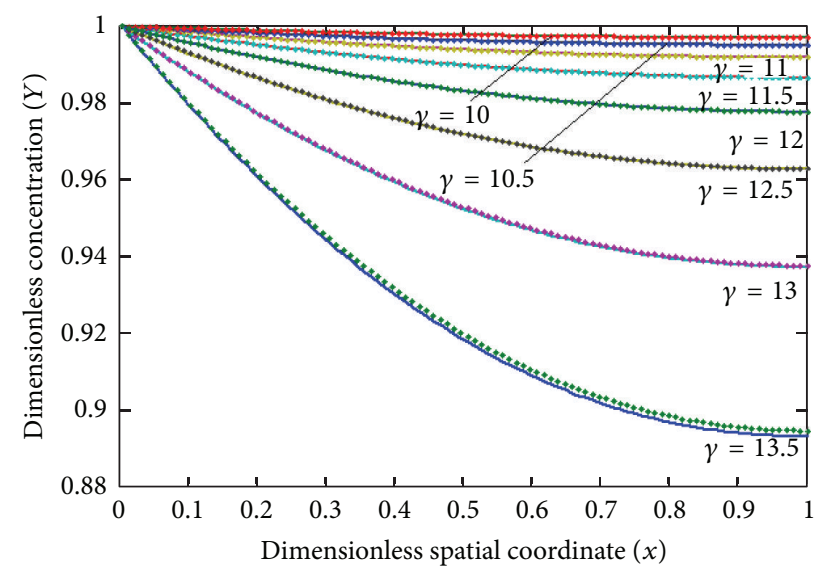

FIGURE 3: Dimensionless concentration $Y$ versus dimensionless spatial coordinate $x$ using (5) for Le $=0.233, \beta=4.287, \phi^{2}=1000$, and various values of $\gamma$. Solid lines represent the analytical solution, whereas the dotted lines are for the numerical solution.

represents the new approximate analytical expression of concentration of gas reactant. The numerical solution is compared with the analytical results in Figures 1-4. These figures represent the analytical and numerical concentration profiles of gas reactant and temperature for different values of parameters $\beta, \gamma$, and $\phi^{2}$. Figure 1 represents the dimensionless concentration $Y$ versus dimensionless spatial coordinate $x$ for $\phi^{2} \leq 35000$. From the figure it is inferred that the value of $Y$ decreases when the value of $\phi^{2}$ or layer thickness increases. Figure 2 illustrates the dimensionless concentration $Y$ versus dimensionless spatial coordinate $x$ for $\phi^{2} \leq 35000$ and we infer that the dimensionless temperature increases with the increase in values of layer thickness. Figure 3 represents the dimensionless concentration $Y$ versus dimensionless spatial coordinate $x$ for values of $\gamma \leq 13.6$. From the figure it is inferred that the value of $Y$ decreases

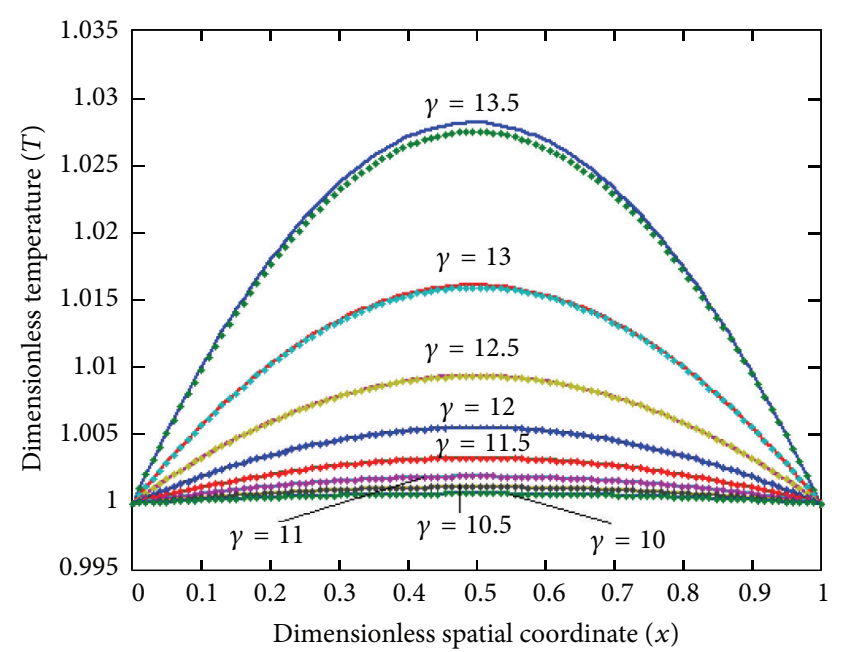

FIgURE 4: Dimensionless temperature $T$ versus dimensionless spatial coordinate $x$ using (6) for Le $=0.233, \beta=4.287 \phi^{2}=1000$, and various values of $\gamma$. Solid lines represent the analytical solution, whereas the dotted lines are for the numerical solution.

TABLE 1: Comparison between analytical and numerical values of dimensionless concentration $Y(\mathrm{Le}=0.233, \beta=4.287, \gamma=13.6$, and $\left.\phi^{2}=30000\right)$.

\begin{tabular}{lccc}
\hline$x$ & Numerical value of $Y$ & Analytical value of $Y$ & \% deviation \\
\hline 0 & 1 & 1 & 0 \\
0.2 & 0.9661 & 0.9713 & 0.53 \\
0.4 & 0.9398 & 0.9489 & 0.97 \\
0.6 & 0.9216 & 0.9329 & 1.23 \\
0.8 & 0.9113 & 0.9233 & 1.32 \\
1 & 0.9082 & 0.9201 & 1.31 \\
\hline
\end{tabular}

when the value of dimensionless activation energy $(\gamma)$ increases. Figure 4 illustrates the dimensionless temperature $T$ versus dimensionless spatial coordinate $x$ for the values of $\gamma \leq 13.6$; the dimensionless temperature increases with increase in values of dimensionless activation energy $(\gamma)$. From Figures 2 and 4 it is evident that the maximum value for dimensionless concentration is 1 and that temperature attains its maximum value when the spatial coordinate $x=$ 0.5 . Figure 5 confirms the results given by Figures 1 to 4.

Our analytical results are compared with the numerical results for the dimensionless concentration $Y$ in Table 1. The maximum relative error between our analytical results and simulation results for the concentration $Y$ is $1.3 \%$. Also in Table 2, our analytical results are compared with the numerical results for the dimensionless temperature $T$. Satisfactory agreement is noted. The maximum relative error in this case is $0.4 \%$.

\section{Conclusion}

The system of steady-state nonlinear differential equations in different dynamic modes for the concentration of 


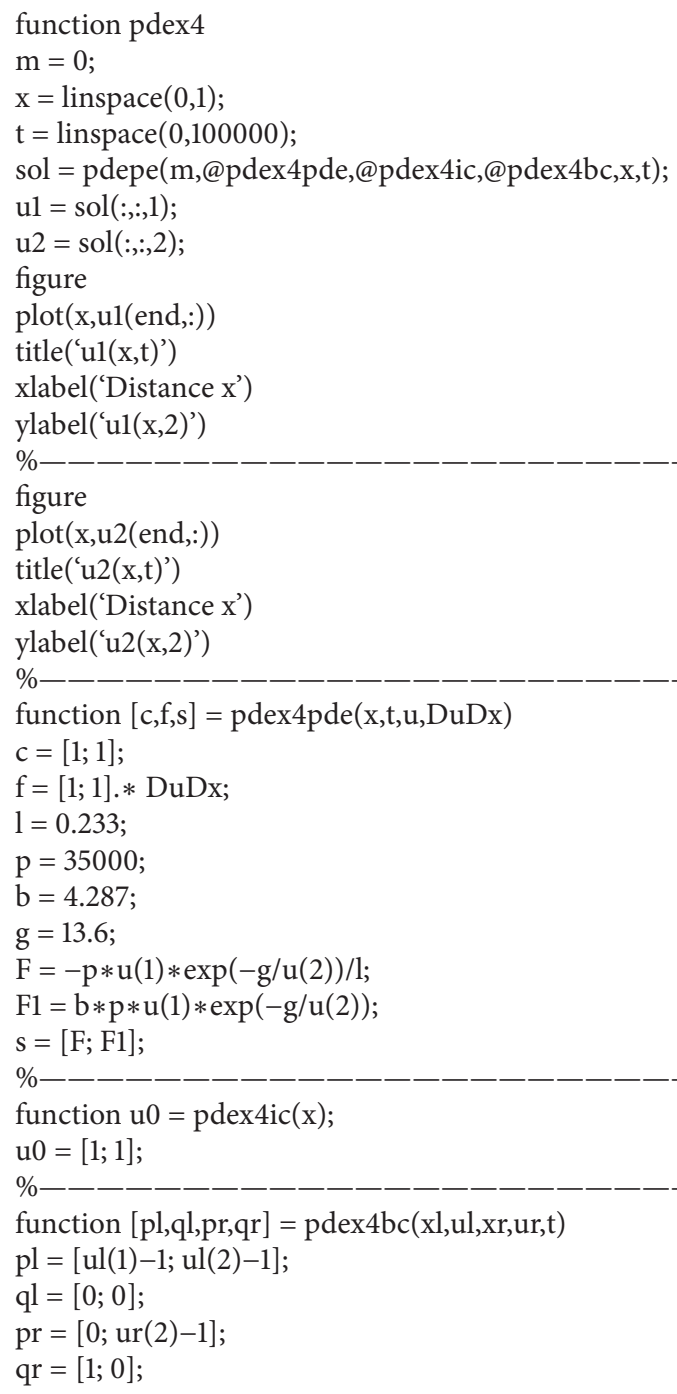

Algorithm 1: MATLAB program to find the numerical solution of nonlinear differential equation (1) when the time $(t)$ is large (or) (3).

TABLE 2: Comparison between analytical and numerical values of dimensionless temperature $T(\mathrm{Le}=0.233, \beta=4.287, \gamma=13.6$, and $\left.\phi^{2}=30000\right)$.

\begin{tabular}{lccc}
\hline$x$ & Analytical value of $T$ & Numerical value of $T$ & $\%$ deviation \\
\hline 0 & 1 & 1 & 0 \\
0.2 & 1.0155 & 1.1013 & 0.27 \\
0.4 & 1.0235 & 1.0191 & 0.42 \\
0.6 & 1.0233 & 1.0191 & 0.41 \\
0.8 & 1.0152 & 1.0128 & 0.24 \\
1 & 1 & 1 & 0 \\
\hline
\end{tabular}

gas reactant and temperature has been solved analytically. The model investigated the influence of parameters over the temperature and concentration of gas reactant in the dynamic mode. The approximate analytical expression for the steady-state concentration of gas reactant and temperature is obtained using the modified Adomian decomposition method. A satisfactory agreement with the numerical result is noted. The analytical results will be useful to characterize the model predictions for the various values of parameters Thiele number $\left(\phi^{2}\right)$, Lewis number (Le), layer thickness $(L)$, and thermal diffusivity $(\alpha)$.

\section{Appendices}

\section{A. Basic Concept of Modified Adomian Decomposition Method}

Consider the nonlinear differential equation in the form

$$
L(y)+N(y)=g(x)
$$

with initial condition

$$
y(0)=A, \quad y^{\prime}(0)=B,
$$




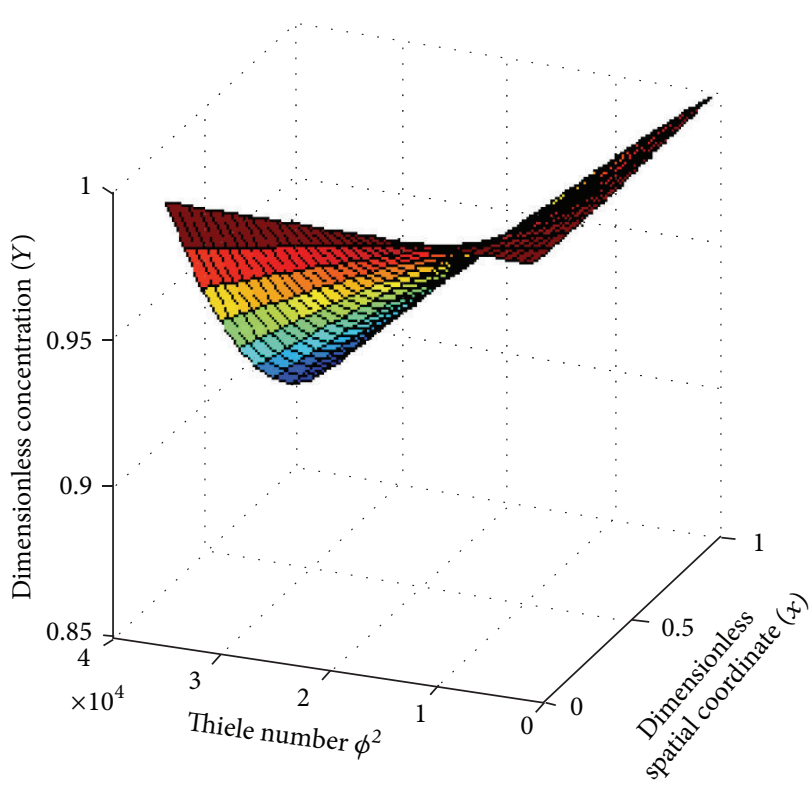

(a)

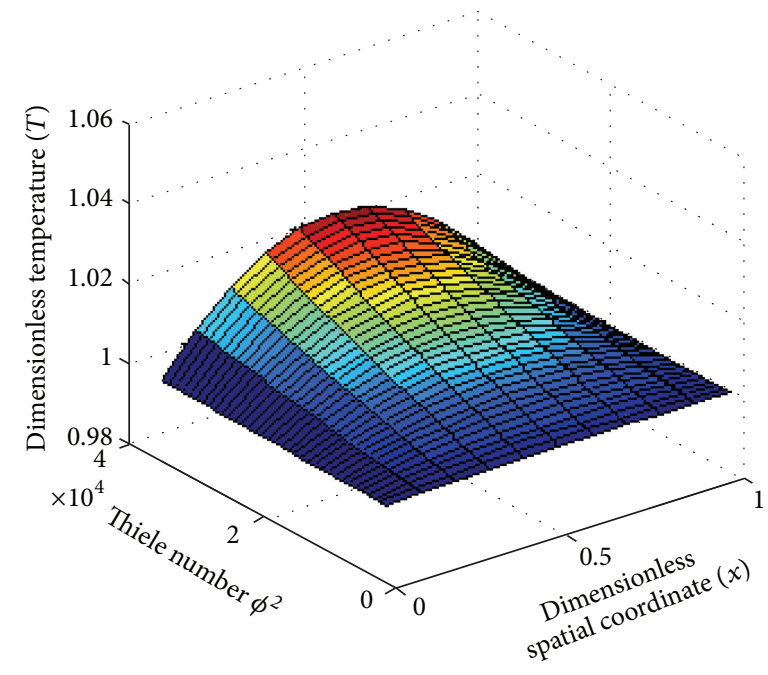

(c)

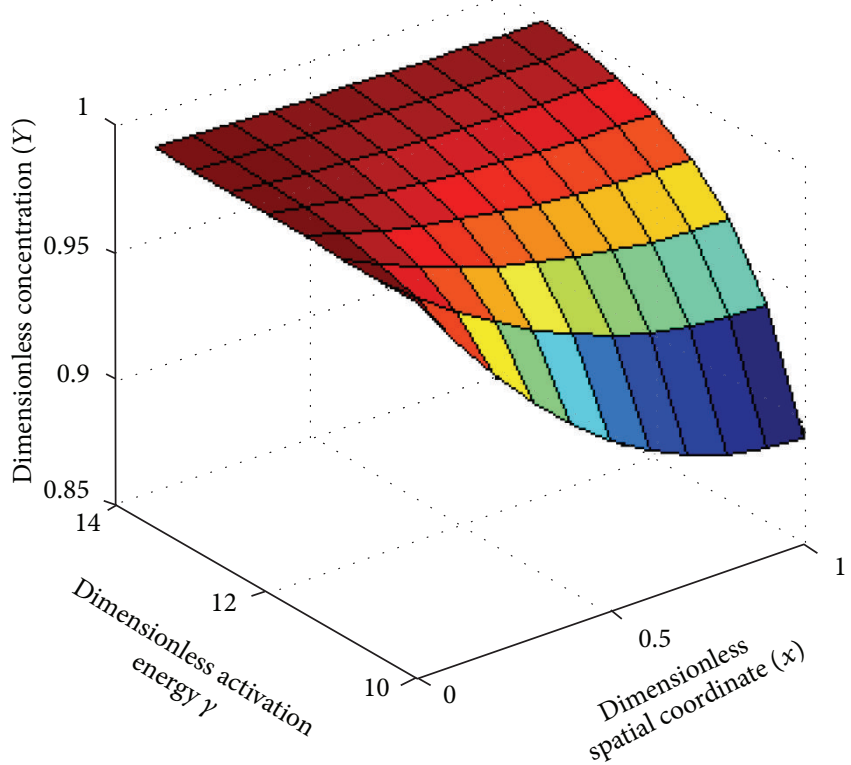

(b)

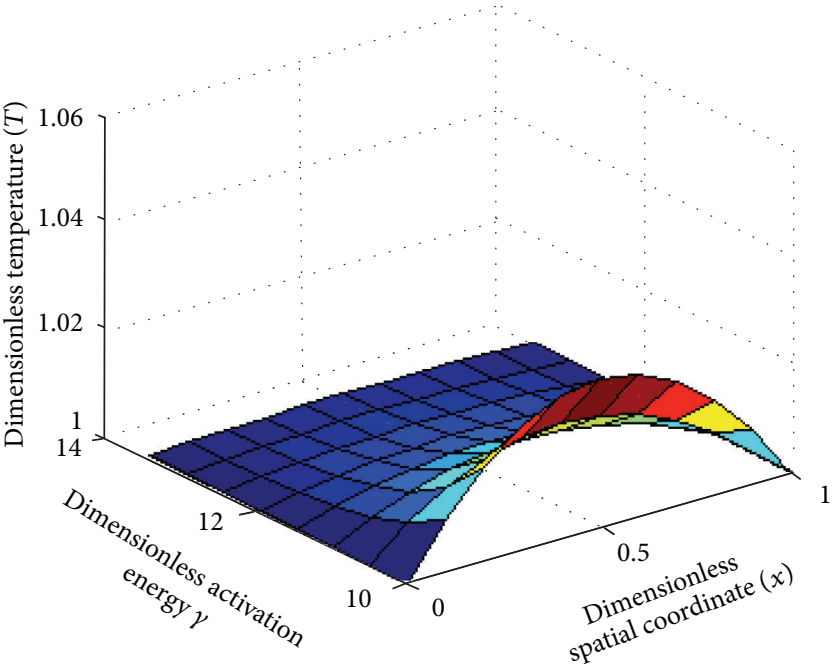

(d)

FIGURE 5: (a) The normalized three-dimensional concentration of gas reactant $Y$ versus dimensionless spatial coordinate $x$ and Thiele number $\phi^{2}$, (b) the normalized three-dimensional concentration of gas reactant $Y$ versus dimensionless spatial coordinate $x$ and dimensionless activation energy $\gamma$, (c) the normalized three-dimensional temperature T versus dimensionless spatial coordinate $x$ and Thiele number $\phi^{2}$, and (d) the normalized three-dimensional temperature $T$ versus dimensionless spatial coordinate $x$ and dimensionless activation energy $\gamma$.

where $N(y)$ is a nonlinear real function, $g(x)$ is the given function, and $A$ and $B$ are constants. We propose the new differential operator as follows:

$$
L=x^{-n} \frac{d^{2}}{d x^{2}} x^{n} y
$$

So, problem (A.1) can be written as

$$
L(y)=g(x)-N(y) .
$$

The inverse operator $L^{-1}$ is therefore considered a twofold integral operator as follows:

$$
L^{-1}(\cdot)=x^{-n} \iint_{0}^{x} x^{n}(\cdot) d x d x
$$

By operating $L^{-1}$ on (A.4), we have

$$
\begin{aligned}
y(x) & =A+B x+L^{-1} g(x)-L^{-1} N(y) \\
& =A+L^{-1} g(x)-L^{-1} N(y) .
\end{aligned}
$$


The modified Adomian decomposition method introduces solution $y(x)$ and the nonlinear function $N(y)$ by infinite series

$$
\begin{gathered}
y(x)=\sum_{n=0}^{\infty} y_{n}(x), \\
N(y)=\sum_{n=0}^{\infty} A_{n},
\end{gathered}
$$

where the components $y_{n}(x)$ of the solution $y(x)$ will be determined recurrently and the Adomian polynomials $A_{n}$ of $F(x, y)$ are evaluated using the formula

$$
A_{n}(x)=\left.\frac{1}{n !} \frac{d^{n}}{d \lambda^{n}} N\left(\sum_{n=0}^{\infty}\left(\lambda^{n} y_{n}\right)\right)\right|_{\lambda=0}
$$

By substituting (A.7) and (A.8) into (A.6),

$$
\sum_{n=0}^{\infty} y_{n}(x)=A+L^{-1} g(x)-L^{-1} \sum_{n=0}^{\infty} A_{n} .
$$

By using the modified Adomian decomposition method, the components $y_{n}(x)$ can be determined as

$$
\begin{aligned}
y_{0}(x) & =A+L^{-1} g(x) y_{n+1}(x) \\
& =-L^{-1}\left(A_{n}\right), \quad n \geq 0,
\end{aligned}
$$

which gives

$$
\begin{aligned}
& y_{0}(x)=A+L^{-1} g(x), \\
& y_{1}(x)=-L^{-1}\left(A_{0}\right), \\
& y_{2}(x)=-L^{-1}\left(A_{1}\right), \\
& y_{3}(x)=-L^{-1}\left(A_{2}\right),
\end{aligned}
$$

From (A.9) and (A.12), we can determine the components $y_{n}(x)$, and hence the series solution of $y(x)$ in (A.7) can be immediately obtained.

\section{B. Analytical Solution of Nonlinear Reaction- Diffusion Equation (3) Using Modified Adomian Decomposition Method}

In this appendix we derive the general solution of nonlinear equation (3) by using modified Adomian decomposition method. We write (3) in the operator form as

$$
\begin{aligned}
& L(Y(x))=k N(Y(x)), \\
& \text { where } L=x^{-1} \frac{d^{2}}{d x^{2}} x \\
& =\frac{\phi^{2}}{\mathrm{Le}} Y \exp \left(-\frac{\gamma}{T}\right), \\
& L(T(x))=k N(T(x)) \\
& =-\beta \phi^{2} Y \exp \left(-\frac{\gamma}{T}\right) .
\end{aligned}
$$

Applying the inverse operator $L^{-1}(\cdot)=x^{-1} \iint_{0}^{x} x(\cdot) d x d x$ on both sides of (B.1) and (B.2) yields

$$
\begin{gathered}
Y(x)=A x+B+L^{-1}\left(\frac{\phi^{2}}{\mathrm{Le}} Y \exp \left(\frac{-\gamma}{T}\right)\right), \\
T(x)=C x+D+L^{-1}\left(-\beta \phi^{2} Y \exp \left(-\frac{\gamma}{T}\right)\right),
\end{gathered}
$$

where $A, B, C$, and $D$ are constants of integration.

Let

$$
\begin{aligned}
& Y(x)=\sum_{n=0}^{\infty} Y_{n}(x), \\
& T(x)=\sum_{n=0}^{\infty} T_{n}(x), \\
& N[Y(x)]=\sum_{n=0}^{\infty} A_{n},
\end{aligned}
$$

where

$$
\begin{gathered}
N[Y(x)]=\frac{\phi^{2}}{\mathrm{Le}} Y \exp \left(-\frac{\gamma}{T}\right), \\
N[T(x)]=\sum_{n=0}^{\infty} B_{n},
\end{gathered}
$$

where

$$
N[T(x)]=-\beta \phi^{2} Y \exp \left(-\frac{\gamma}{T}\right) .
$$

In view of (B.5), (B.7), and (B.8), (B.3) gives

$$
\sum_{n=0}^{\infty} Y_{n}(x)=A x+B+L^{-1}\left(\sum_{n=0}^{\infty} A_{n}\right) .
$$

We identify the zeroth component as

$$
Y_{0}(x)=A x+B .
$$


Using initial condition (4), we get $A=0$ and $B=1$;

$$
\therefore Y_{0}=1
$$

and the remaining components are as the recurrence relation

$$
Y_{n+1}(x)=L^{-1}\left(A_{n}\right), \quad n \geq 0,
$$

where $A_{n}$ are the Adomian polynomials of $Y_{1}, Y_{2}, \ldots Y_{n}$. We can find $A_{n}$ as follows:

$$
\begin{gathered}
A_{0}=N\left(Y_{0}\right), \\
A_{1}=\frac{d}{d \lambda} N\left(Y_{0}+\lambda Y_{1}\right) .
\end{gathered}
$$

In view of (B.6), (B.9), and (B.10), (B.4) gives

$$
\sum_{n=0}^{\infty} T_{n}(x)=C x+D+L^{-1}\left(\sum_{n=0}^{\infty} B_{n}\right) .
$$

We identify the zeroth component as

$$
T_{0}(x)=C x+D .
$$

Using initial condition (4), we get $C=0$ and $D=1$;

$$
\therefore T_{0}=1
$$

and the remaining components are as the recurrence relation

$$
T_{n+1}(x)=L^{-1}\left(B_{n}\right), \quad n \geq 0,
$$

where $B_{n}$ are the Adomian polynomials of $T_{1}, T_{2}, \ldots, T_{n}$. We can find $B_{n}$ as follows:

$$
\begin{gathered}
B_{0}=N\left(T_{0}\right) . \\
B_{1}=\frac{d}{d \lambda} N\left(T_{0}+\lambda T_{1}\right) .
\end{gathered}
$$

Hence solving (B.14) and (B.19) we get the following solutions:

$$
\begin{gathered}
Y_{1}=\frac{\phi^{2}}{\mathrm{Le}} \exp (-\gamma)\left(\frac{x^{2}}{2}-x\right), \\
T_{1}=-\beta \phi^{2} \exp (-\gamma)\left(\frac{x^{2}}{2}-\frac{x}{2}\right), \\
Y_{2}=\frac{\phi^{4}}{\mathrm{Le}} \exp (-2 \gamma)\left(\frac{1}{\mathrm{Le}}\left(\frac{x^{4}}{24}-\frac{x^{3}}{6}+\frac{x}{3}\right)\right. \\
\left.-\frac{\gamma \beta}{2}\left(\frac{x^{4}}{12}-\frac{x^{3}}{6}+\frac{x}{6}\right)\right), \\
T_{2}=-\beta \phi^{4} \exp (-2 \gamma)\left(\frac{1}{\operatorname{Le}}\left(\frac{x^{4}}{24}-\frac{x^{3}}{6}+\frac{x}{8}\right)\right. \\
\left.-\frac{\gamma \beta}{2}\left(\frac{x^{4}}{12}-\frac{x^{3}}{6}+\frac{x}{12}\right)\right) .
\end{gathered}
$$

Adding (B.13), (B.21), and (B.23) we get (5) in the text. Adding (B.18), (B.22), and (B.24) we get (6) in the text.
C.

See Algorithm 1.

\section{Notations}

Symbols

$c_{0}$ : Concentration of the free stream

$c_{p}:$ Specific heat

D: Mass diffusivity

E: Activation energy

$K_{0}$ : Preexponential factor

$L:$ layer thickness

Le: Lewis number, $D / \alpha$

$R:$ Gas reactant

$T$ : Dimensionless temperature $\widetilde{T} / \widetilde{T}_{0}$

$\widetilde{T}$ : Temperature

$\widetilde{T}_{0}$ : Free stream temperature

$t$ : Dimensionless time $\tilde{t} \alpha / L^{2}$

$\tilde{t}:$ Time

$Y:$ Dimensionless concentration $c / c_{0}$.

Greek letters

$\alpha$ : Thermal diffusivity

$\beta: \quad$ Dimensionless heat reaction $\left(-\Delta H c_{0}\right) /\left(\rho c_{p} \widetilde{T}_{0}\right)$

$\gamma: \quad$ Dimensionless activation energy, $E /\left(R \widetilde{T}_{0}\right)$

$\Delta H$ : Enthalpy of reaction

$\rho:$ Density

$\Phi: \quad$ Thiele number, $\sqrt{k_{0} L^{2} / \alpha}$.

\section{Conflict of Interests}

The authors declare that there is no conflict of interests regarding the publication of this paper.

\section{Acknowledgments}

This work is supported by the University Grant Commission (UGC) Minor Project no. F. MRP-4122/12 (MRP/UGCSERO), Hyderabad, Government of India. The authors are thankful to Shri. S. Natanagopal, secretary at Madura College Board, and Dr. R. Murali, principal at Madura College (autonomous), Madurai, Tamil Nadu, India, for their constant encouragement.

\section{References}

[1] G. Continillo, V. Faraoni, P. L. Maffettone, and S. Crescitelli, "Non-linear dynamics of a self-igniting reaction-diffusion system," Chemical Engineering Science, vol. 55, no. 2, pp. 303-309, 2000.

[2] G. Continillo, P. L. Maffettone, and S. Crescitelli, "On the numerical simulation of the chaotic behavior of some distributed-parameter systems," in Proceedings of the 1st National 
Conference Chaos and Fractals in Chemical Engineering, pp. 218226, World Scientific, Singapore, May 1994.

[3] C. Gaetano and G. Giovanni, Characterization of Chaotic Dynamics in the Spontaneous Combustion of Coal Stockpiles, Istituto di Ricerchesulla Combustione (IRC) Consiglio Nazionaledelle Ricerche, Naples, Italy, 1996.

[4] G. Continillo, P. L. Maffettone, and S. Crescitelli, First Conference on Chemical and Process Engineering, Paper no. 2.2, Firenze, Italy, 1993.

[5] G. Continillo, P. L. Maffettone, and S. Crescitelli, "On the numerical simulation of the chaotic behaviour of some distributed-parameter systems," in Chaos and Fractals in Chemical Engineering, G. Biardi, M. Giona, and A. R. Giona, Eds., pp. 218-226, World Scientific, Singapore, 1995.

[6] G. Continillo, P. L. Maffettone, and S. Crescitelli, in ICheaP-2, C. T. Eris, Ed., vol. 1 of AIDIC Conference Series, pp. 415-424, Firenze, Italy, 1995.

[7] W. B. J. Zimmerman, Microfluidics, History Theory \& Applications, Springer, New York, NY, USA, 2006.

[8] G. Adomian, "Convergent series solution of nonlinear equations," Journal of Computational and Applied Mathematics, vol. 11, no. 2, pp. 225-230, 1984.

[9] Y. Q. Hasan and L. M. Zhu, "Modified Adomian decomposition method for singular initial value problems in the second-order ordinary differential equations," Surveys in Mathematics and Its Applications, vol. 3, pp. 183-193, 2008.

[10] Y. Q. Hasan and L. M. Zhu, "Solving singular boundary value problems of higher-order ordinary differential equations by modified Adomian decomposition method," Communications in Nonlinear Science and Numerical Simulation, vol. 14, no. 6, pp. 2592-2596, 2009.

[11] A.-M. Wazwaz, "A reliable modification of Adomian decomposition method," Applied Mathematics and Computation, vol. 102, no. 1, pp. 77-86, 1999.

[12] A.-M. Wazwaz, "Analytical approximations and pade approximants for volterra's population model," Applied Mathematics and Computation, vol. 100, no. 1, pp. 13-25, 1999.

[13] A.-M. Wazwaz, "A new method for solving singular initial value problems in the second-order ordinary differential equations," Applied Mathematics and Computation, vol. 128, no. 1, pp. 45-57, 2002. 

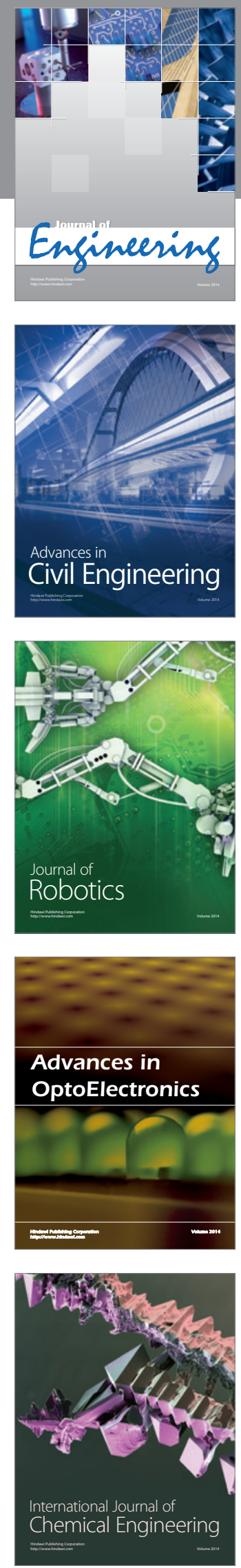

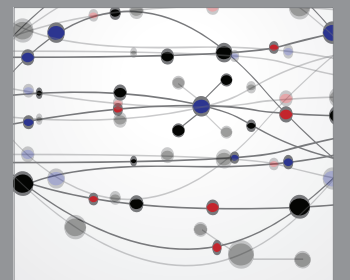

The Scientific World Journal
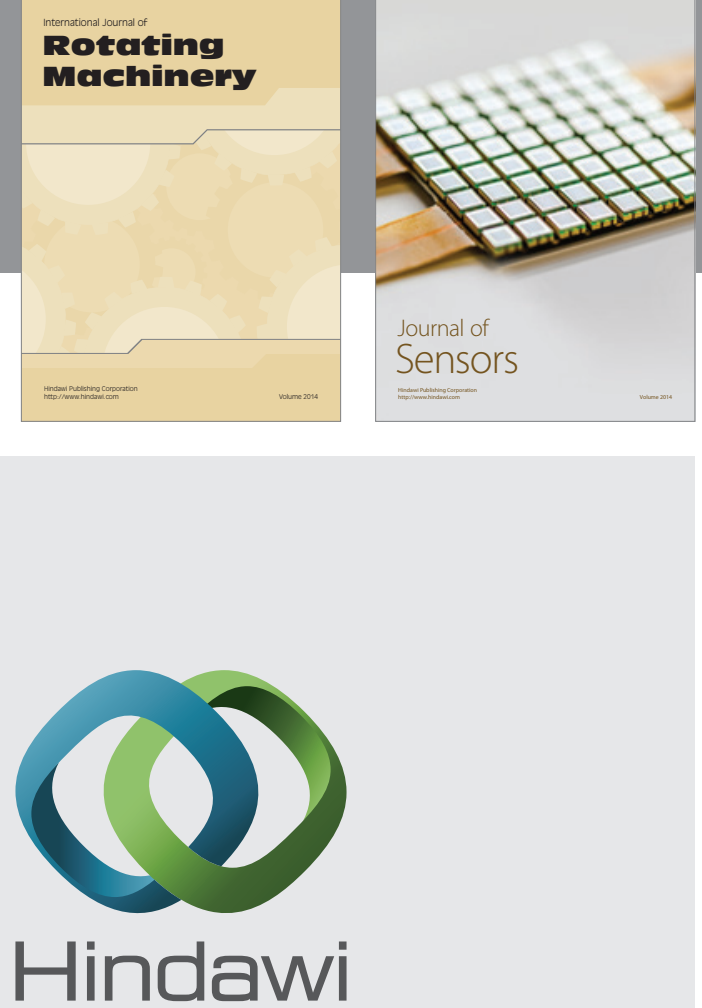

Submit your manuscripts at http://www.hindawi.com
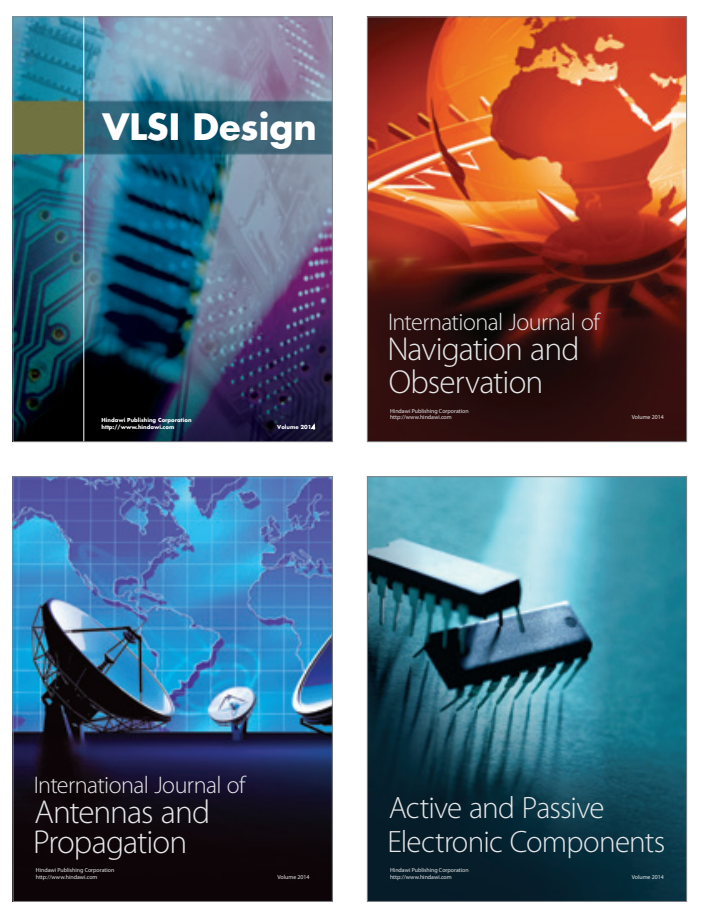
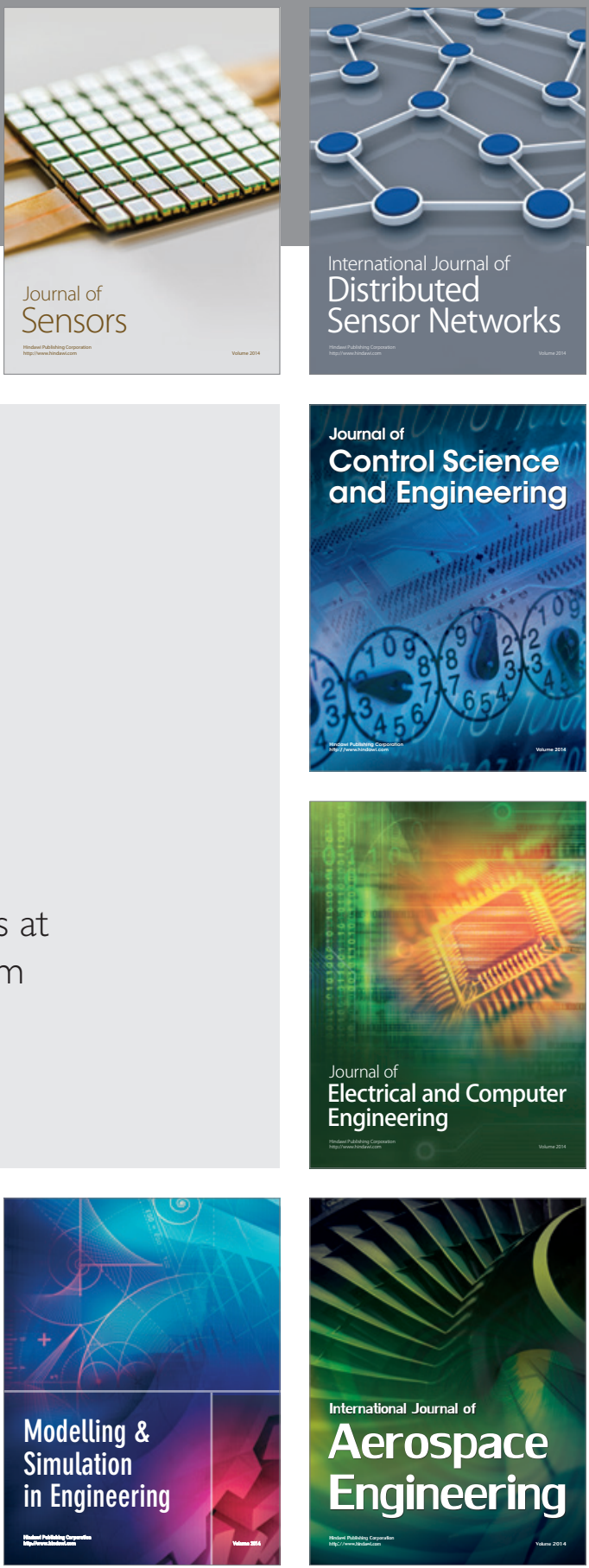

Journal of

Control Science

and Engineering
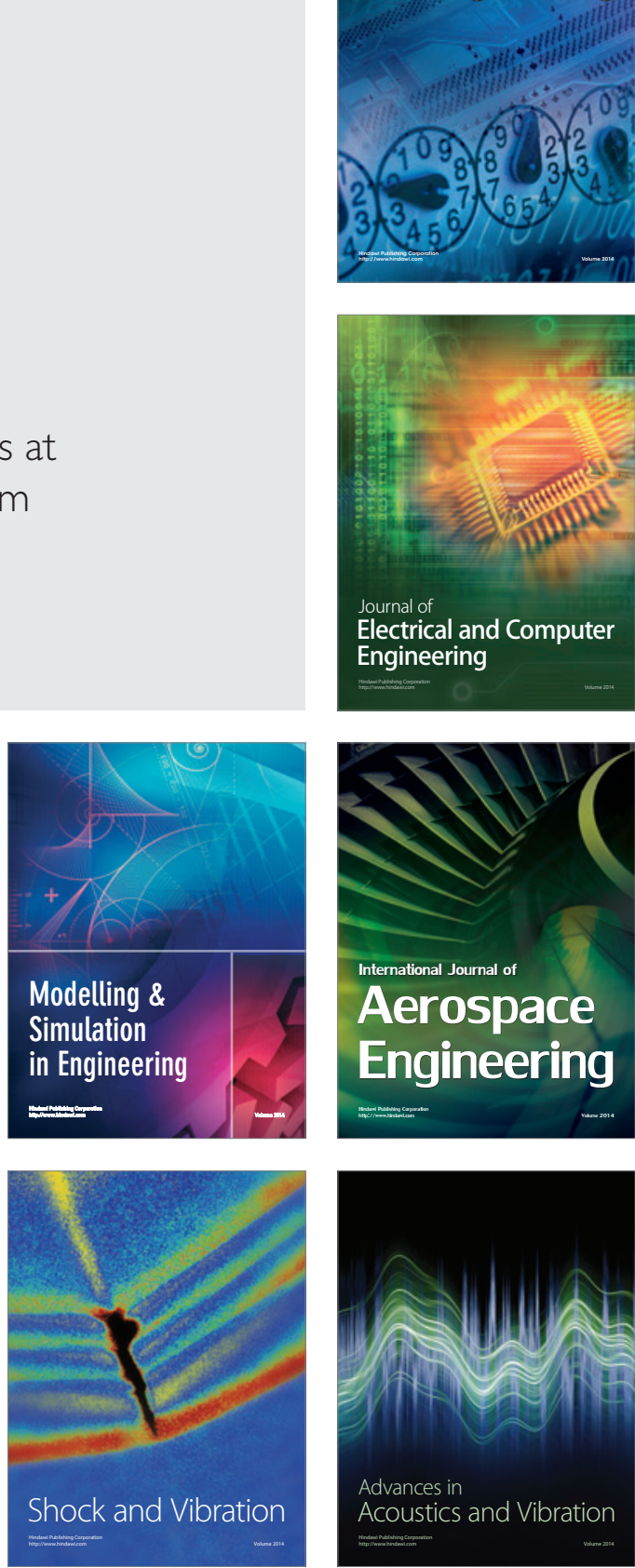